\title{
EFECTO DE HERBICIDAS SOBRE PLANTAS Y SEMILLAS DE Rottboellia cochinchinensis (Lour.) W. Clayton, EN CAÑA DE AZÚCAR1
}

\author{
Valentín A. Esqueda Esquivel ${ }^{2}$
}

\begin{abstract}
RESUMEN
Efecto de herbicidas sobre plantas y semillas de Rottboellia cochinchinensis (Lour.) W. Clayton, en caña de azúcar. Se evaluó la efectividad de herbicidas que se utilizan comúnmente en caña de azúcar en el control del zacate peludo (Rottboellia cochinchinensis) y su banco de semillas del suelo. En un lote sembrado con caña de azúcar de la variedad MEX 69-290 en Cosamaloapan, Veracruz, México en junio del 2000, se evaluaron nueve tratamientos: 1 . Tebuthiurón (1500 g/ha), 2. Diurón/hexazinona (1634,4/204,3 g/ha), 3. Diurón (2400 g/ha), 4. Ametrina/2,4-D (1225/650 g/ha) 5. Ametrina/2,4-D + Diurón/hexazinona (735/390 + 544,8/68,1 g/ha), 6 . Tebuthiurón + Diurón/hexazinona $(1.000+817,2 / 102,2 \mathrm{~g} / \mathrm{ha})$, 7. Tebuthiurón + Diurón $(1.000+1.200 \mathrm{~g} / \mathrm{ha}), 8$. Tebuthiurón + Ametrina/2,4-D (1.000 + 490/260 g/ha) y 9. Testigo sin aplicar. Se utilizó un diseño experimental de bloques al azar con cuatro repeticiones. El control del zacate peludo fue evaluado a los 15, 30, 60 y 90 días después de la aplicación (DDA). Se tomaron muestras de suelo (0-20 cm de profundidad) a los 0 , $30,90,120$ y 150 DDA. El suelo extraído de cada parcela experimental fue puesto en una charola y regado diariamente. Se contabilizó el número de plántulas de zacate peludo emergidas durante 75 días. El mejor control del zacate peludo se obtuvo con tebuthiurón a $1.500 \mathrm{~g} / \mathrm{ha}$; un buen control también fue obtenido con tebuthiurón + diurón a $1.000+1.200 \mathrm{~g} /$ ha y tebuthiurón + diurón/hexazinona a $1.000+817,2 / 102,2 \mathrm{~g} / \mathrm{ha}$. Todos los herbicidas tuvieron una reducción significativa en la emergencia de plántulas de zacate peludo, que varió de 59 a $96 \%$, comparada con la del testigo sin aplicar.
\end{abstract}

Palabras clave: herbicidas, Rottboellia cochinchinensis, caña de azúcar, plantas, semillas.

\begin{abstract}
Effect of herbicides on plants and seeds of itchgrass (Rottboellia cochinchinensis (Lour.) W. Clayton) in sugarcane. An experiment was carried out to evaluate the effectiveness of several herbicides commonly used in sugarcane on the control of itchgrass (Rottboellia cochinchinensis) and their effect on its seed bank. The experiment was established in a plot sown with the sugarcane variety MEX 69-290 in Cosamaloapan, Veracruz, Mexico, during the 2000 rainy season. Nine treatments were evaluated: 1 . Tebuthiuron (1500 g/ha), 2. Diuron/hexazinone (1634.4/204.3 g/ha), 3. Diuron (2400 g/ha), 4. Ametrine/2,4D (1225/650 g/ha) 5. Ametrine/2,4-D + Diuron/hexazinone $(735 / 390+544.8 / 68.1 \mathrm{~g} / \mathrm{ha}), 6$. Tebuthiuron + Diuron/hexazinone $(1000+817.2 / 102.2 \mathrm{~g} / \mathrm{ha}), \quad 7$. Tebuthiuron + Diuron $(1000+1200 \mathrm{~g} / \mathrm{ha}), 8$. Tebuthiuron + Ametrine/2,4-D (1000 + 490/260 g/ha) and 9. Weedy control. A Randomized Complete Block Design with four replications was utilized. Itchgrass control was evaluated at 15, 30, 60 and 90 days after application (DAA). Soil samples $(0-20 \mathrm{~cm}$ deep) were taken at $0,30,90,120$ and 150 DAA. The soil from each experimental plot was put in a tray and watered daily. Itchgrass seedlings that emerged during 75 days were counted. The best itchgrass control was obtained with tebuthiuron at $1500 \mathrm{~g} / \mathrm{ha}$; a good control was also obtained with tebuthiuron + diuron at $1000+1200 \mathrm{~g} / \mathrm{ha}$ and tebuthiuron + diuron/hexazinone at $1000+817.2 / 102.2 \mathrm{~g} / \mathrm{ha}$. All herbicide treatments had a significant reduction in the emergence of itchgrass seedlings which varied from 59 to $96 \%$, compared with the weedy control.
\end{abstract}

Key words: herbicides, Rottboellia cochinchinensis, sugarcane, plants, seeds.

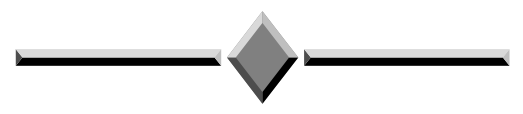

\section{INTRODUCCIÓN}

El zacate peludo (Rottboellia cochinchinensis (Lour.) W. Clayton), anteriormente conocido como $R$. exaltata L. f., es una gramínea anual originaria del su- reste asiático, de crecimiento rápido y altamente competitivo con los cultivos (Lencse y Griffin 1991; Millhollon y Burner 1993). Durante su ciclo de vida, una planta, es capaz de producir hasta 15.000 semillas (Rojas et al. 1994), las cuales se desprenden con mucha

\footnotetext{
${ }^{1}$ Recibido: 26 de mayo, 2004. Aceptado: 16 de diciembre, 2004 .

${ }^{2}$ Campo Experimental Cotaxtla, México. CIRGOC. INIFAP. SAGARPA. esqueda.valentin@inifap.gob.mx
} 
facilidad y si son enterradas, mantienen su viabilidad por tres o cuatro años (León y Agüero 2001). Esta especie fue colectada por primera vez en México, en la zona de Escárcega, Campeche, en 1982 y posteriormente fue detectada en terrenos cañeros del área de Tuxtepec, Oaxaca, en 1984 (Gómez 1985). En la actualidad, el zacate peludo está ampliamente distribuído en los estados colindantes con el Golfo de México, en los cultivos de arroz de temporal (Esqueda 2000b), plátano (Castillo et al. 1991), maíz (Tucuch 1991), cítricos (Medina y Domínguez 2001) y caña de azúcar (Esqueda 1999), en donde ha llegado a convertirse en la especie dominante en la región de la Cuenca Baja del Río Papaloapan.

Los herbicidas formulados con ametrina y diurón, que se usan ampliamente en caña de azúcar, son inconsistentes en el control del zacate peludo (Esqueda 1999). Existe un herbicida formulado con tebuthiurón, que cuando se aplica en preemergencia, controla eficientemente las principales especies de malezas anuales de este cultivo, por más de cuatro meses (Esqueda 1997). También puede mezclarse con otros herbicidas formulados con hexazinona, ametrina y diurón y aplicarse en postemergencia temprana, lo cual sirve para ampliar el espectro de malezas controladas y evitar problemas de poca humedad del terreno, que son frecuentes al inicio de las lluvias y que pueden reducir su efectividad (Esqueda 2000a).

Debido a que la residualidad del tebuthiurón es mayor a la de los otros herbicidas que se utilizan en el cultivo de caña de azúcar, se considera, que este producto sólo, o en mezcla con otros herbicidas, puede ayudar a controlar al zacate peludo y reducir su banco de semillas en los terrenos cañeros, en que se utilice por varios años. Asimismo, debe observarse su efecto en otras especies de malezas de hoja ancha, que también se presentan con frecuencia en la caña de azúcar (Morales 1987).

El objetivo de esta investigación fue determinar el efecto de herbicidas residuales en el control del zacate peludo y otras malezas de hoja ancha en caña de azúcar. Evaluar el efecto residual de estos herbicidas en la viabilidad del banco de semillas del zacate peludo, y evaluar el efecto fitotóxico de los herbicidas en la caña de azúcar.

\section{MATERIALES Y MÉTODOS}

Se estableció un experimento en una parcela con socas de caña de azúcar de la variedad MEX 69-290, sembrada a una densidad de $12.000 \mathrm{~kg} / \mathrm{ha}$, en el Ejido Gabino Barreda, municipio de Cosamaloapan, Veracruz, México. Se evaluaron nueve tratamientos incluyendo un testigo sin aplicar (Cuadro 1).
Cuadro 1. Descripción de los tratamientos. Gabino Barreda, mpio. Cosamaloapan, Veracruz, México. 2000.

\begin{tabular}{clc}
\hline No. & \multicolumn{1}{c}{ Tratamiento* } & Dosis (g/ha) \\
\hline & Tebuthiurón & 1.500 \\
2 & Diurón/hexazinona & $1.634,4 / 204,3$ \\
3 & Diurón & 2.400 \\
4 & Ametrina/2,4-D & $1.225 / 650$ \\
5 & Ametrina/2,4-D + diurón/ & \\
& hexazinona & $735 / 390+544,8 / 68,1$ \\
6 & Tebuthiurón + diurón/hexazinona & $1.000+817,2 / 102,2$ \\
7 & Tebuthiurón + diurón & $1.000+1.200$ \\
8 & Tebuthiurón + ametrina/2,4-D & $1.000+490 / 260$ \\
9 & Testigo sin aplicar & - \\
\hline
\end{tabular}

* Cuando dos ingredientes activos están separados por “/”, representa una mezcla formulada de fábrica. Cuando dos ingredientes activos están separados por “+”, representa una mezcla de tanque.

Se utilizó un diseño experimental de bloques completos al azar con cuatro repeticiones. Cada parcela experimental estuvo constituida por ocho surcos de $10 \mathrm{~m}$ de longitud y 1,40 m de separación, lo que representó una superficie de $112 \mathrm{~m}^{2}$. Los herbicidas se aplicaron con una aspersora motorizada de mochila, a la cual se le adaptó un aguilón con cuatro boquillas XR Tee jet 11005 $\mathrm{VP}$, que proporcionó un volumen de aspersión equivalente a 154 1/ha. El tratamiento uno se aplicó el 1 de junio del 2000, en preemergencia a las malezas y cuando la caña de azúcar tenía entre 30 y $40 \mathrm{~cm}$ de altura. A su vez, los tratamientos del dos al ocho se aplicaron el 22 de junio del 2000, cuando la caña tenía una altura de entre 60 y $90 \mathrm{~cm}$ y el zacate peludo entre 10 y $60 \mathrm{~cm}$. Se añadió el surfactante no iónico Agral Plus en dosis de $250 \mathrm{ml}$ por cada 1001 de solución. Sólo se aplicaron los seis surcos centrales de cada parcela experimental y se permitió el desarrollo de la maleza en los dos surcos laterales, con la finalidad de utilizarlos como testigos laterales enhierbados, al momento de las evaluaciones de control. En las parcelas correspondientes al testigo sin aplicar, se permitió el libre desarrollo de la maleza durante el período de conducción del experimento.

El cultivo se desarrolló en condiciones de temporal. La caña de azúcar se fertilizó al inicio de las lluvias con $400 \mathrm{~kg}$ de la fórmula 17-17-17/ha. No fue necesario el uso de insecticidas ni fungicidas durante el tiempo que se condujo el experimento.

La densidad de población de malezas se determinó al momento de la aplicación de los tratamientos postemergentes y a los 15 y 30 días después de ésta. Para esto, se utilizó un cuadrante de 1 x $1 \mathrm{~m}$, el cual se lanzó al azar en dos ocasiones en cada una de las parcelas 
correspondientes a los testigos sin aplicar. Las malezas contenidas en el interior de los cuadrantes, fueron identificadas y cuantificadas y se hicieron las transformaciones necesarias para determinar su densidad de población por hectárea.

Las evaluaciones de control del zacate peludo y el complejo de malezas de hoja ancha se llevaron a cabo a $\operatorname{los} 15,30,60$ y 90 días después de la aplicación (DDA). Se evaluó visualmente el efecto de los herbicidas en la totalidad de cada parcela experimental. Para evaluar se utilizó la escala porcentual (0-100\%), en donde 0 , significó que las malezas no fueron afectadas y 100 , que fueron completamente eliminadas. La evaluación visual del control de malezas mediante la escala porcentual es ampliamente utilizada, ejemplos de ello en caña de azúcar se tienen en Sánchez y Zárate (2000), Esqueda (1997) y Esqueda (1999).

Para determinar el efecto residual de los tratamientos en la emergencia de plántulas de zacate peludo, en cada parcela experimental se tomaron dos muestras de suelo al azar, con un cilindro de $14 \mathrm{~cm}$ de diámetro y 20 cm de profundidad a los 0, 30, 90, 120 y 150 DDA. El suelo obtenido de los dos puntos muestreados en cada parcela experimental, se mezcló y se tomaron muestras de $1,5 \mathrm{~kg}$ que se llevaron al Campo Experimental Cotaxtla del INIFAP, en donde se colocaron en charolas de plástico de $23,5 \mathrm{~cm}$ de largo x $19 \mathrm{~cm}$ de ancho y $10 \mathrm{~cm}$ de profundidad. Las charolas se regaron diariamente y durante 75 días se evaluó la emergencia de plántulas de zacate peludo, las cuales fueron arrancadas después de ser contabilizadas.

Se evaluó visualmente la toxicidad de los tratamientos herbicidas a la caña de azúcar, a los 15 y 30
DDA, con base en la escala de 0 a $100 \%$, en donde 0 significó que la caña no fue afectada y $100 \%$ que fue completamente destruída, como se indica en Altamirano et al. (2000).

Para homogenizar las varianzas, los datos experimentales de control de zacate peludo y malezas de ho$\mathrm{ja}$ ancha fueron transformados a su valor de arco seno $\mathrm{x}$ $\sqrt{\%}$ y los de toxicidad a la caña de azúcar, a su valor de $\sqrt{\%}$, de acuerdo a lo que se recomienda en Gomez y Gomez (1984). Como prueba de separación de promedios se utilizó Tukey $(0,05)$. Aún cuando los análisis de varianza se realizaron con datos transformados, por motivos de claridad, se presentan los promedios de los datos de campo.

\section{RESULTADOS Y DISCUSIÓN}

En el sitio experimental se presentaron nueve especies de malezas, pertenecientes a seis familias botánicas. Siete de las especies encontradas tienen un ciclo de vida anual y en dos el ciclo de vida es perenne (Cuadro 2). Al momento de la aplicación de los tratamientos postemergentes, la población total de malezas era de 605.000 plantas/ha. A los 15 DDA, se observó un incremento en el número de malezas emergidas hasta llegar a 1070.000 plantas/ha, para finalmente disminuir a 735.000 plantas/ha a los 30 DDA. La especie dominante fue $R$. cochinchinensis, que ocupó entre el 42,2 y $71,1 \%$ de la población de malezas, en los diferentes conteos (Cuadro 3).

Tebuthiurón fue el tratamiento que mostró el mayor control del zacate peludo en las distintas épocas de evaluación. Hasta los 30 DDA, este herbicida ofreció un

Cuadro 2. Especies de malezas presentes en el sitio experimental. Gabino Barreda, municipio Cosamaloapan, Veracruz, México. 2000.

\begin{tabular}{|c|c|c|c|}
\hline Nombre común & Nombre científico & Familia* & Ciclo de vida \\
\hline Zacate peludo & $\begin{array}{l}\text { Rottboellia cochinchinensis } \\
\text { (Lour.) W. Clayton }\end{array}$ & Poaceae & Anual \\
\hline Papayita & Croton lobatus $\mathrm{L}$. & Euphorbiaceae & Anual \\
\hline Zacate carricillo & $\begin{array}{l}\text { Urochloa fasciculata (Sw.) R. } \\
\text { Webster }\end{array}$ & Poaceae & Anual \\
\hline Frente de toro & Digitaria ciliaris (Retz.) Koel. & Poaceae & Anual \\
\hline Coquillo & Cyperus rotundus $\mathrm{L}$. & Cyperaceae & Perenne \\
\hline Mafafa & Colocasia sp. & Araceae & Perenne \\
\hline Gusanillo & Acalypha alopecuroides Jacq. & Euphorbiaceae & Anual \\
\hline Cundeamor & Momordica charantia L. & Cucurbitaceae & Anual \\
\hline Quelite & Amaranthus sp. & Amaranthaceae & Anual \\
\hline
\end{tabular}

* Se utilizó el nombre de la familia botánica de acuerdo a Villaseñor y Espinosa (1998). 
Cuadro 3. Densidad de población de malezas (plantas/ha) a los 0, 15 y 30 DDA. Gabino Barreda, municipio Cosamaloapan, Veracruz, México. 2000.

\begin{tabular}{lrrr}
\hline \multicolumn{1}{c}{ Especie } & O DDA & 15 DDA & 30 DDA \\
\hline R. cochinchinensis & 430.000 & 715.000 & 310.000 \\
C. lobatus & 60.000 & 85.000 & 60.000 \\
U. fasciculata & 35.000 & 65.000 & 55.000 \\
D. ciliaris & 20.000 & 20.000 & 30.000 \\
C. rotundus & 15.000 & 25.000 & 65.000 \\
Colocasia sp. & 15.000 & 55.000 & 65.000 \\
A. alopecuroides & 15.000 & 45.000 & 70.000 \\
M. charantia & 10.000 & 35.000 & 65.000 \\
Amaranthus sp. & 5.000 & 25.000 & 15.000 \\
Total & 605.000 & 1070.000 & 735.000 \\
\hline
\end{tabular}

control total de esta especie y éste se mantuvo por arriba del $90 \%$ hasta los 90 DDA. La mezcla formulada de diurón/hexazinona mantuvo un control entre 85 y $90 \%$ hasta los 30 DDA, pero a partir de los 60 DDA, el control osciló entre el 70 y $75 \%$. El diurón tuvo un control superior al 95\% hasta los 30 DDA y se redujo paulatinamente hasta quedar en $80 \%$ a los 90 DDA. Desde un inicio, el control del zacate peludo por parte de la mezcla formulada de ametrina/2,4-D fue de solamente $64 \%$, para terminar en 35\% a los 90 DDA, lo que concuerda con lo encontrado por Esqueda 1999. En los tratamientos con las mezclas de tanque de herbicidas, los mejores controles del zacate peludo se obtuvieron con tebuthiurón + diurón/hexazinona y tebuthiurón + diurón. En ambas mezclas, los controles se mantuvieron por arriba del $90 \%$ hasta los 30 DDA, del $85 \%$ hasta los 60 DDA y del $80 \%$ hasta los 90 DDA. A su vez, las mezclas de ametrina/2,4$\mathrm{D}+$ diurón/hexazinona y de tebuthiurón + ametrina/2,4D tuvieron controles de entre 80 y $90 \%$ hasta los 30 DDA, y disminuyeron a $70 \%$ a los 60 DDA, para terminar entre 60 y $65 \%$ a los 90 DDA (Cuadro 4).

Se observó un control total de las distintas especies de maleza de hoja ancha hasta los 30 DDA. Entre los 30 y 60 DDA, todos los tratamientos herbicidas mostraban controles de las hojas anchas superiores al 90\%, con excepción de ametrina/2,4-D, cuyo control fue de $88 \%$. Finalmente, a los 90 DDA, ametrina/2,4-D y diurón mostraban controles de las malezas de hoja ancha entre 80 y $90 \%$, mientras que en el resto de los tratamientos el control era superior al 90\%, destacando tebuthiurón, con 98\% (Cuadro 5).

El número de plántulas de zacate peludo que se contabilizaron en las charolas que contenían la tierra del muestreo efectuado antes de aplicar los tratamientos herbicidas, varió entre 5 y 8,75 , sin existir diferencias
Cuadro 4. Efecto de los tratamientos en el control de zacate peludo (\%) a los 15, 30, 60 y 90 DDA. Gabino Barreda, municipio Cosamaloapan, Veracruz, México. 2000.

\begin{tabular}{|c|c|c|c|c|}
\hline Tratamientos (dosis g/ha) 1 & 15 DDA & 30 DDA & 60 DDA & 90 DDA \\
\hline Tebuthiurón (1.500) & $100 a^{*}$ & $100 \mathrm{a}$ & $98 \mathrm{a}$ & $93 \mathrm{a}$ \\
\hline \multicolumn{5}{|l|}{ Diurón/hexazinona } \\
\hline$(1634,4 / 204,3)$ & $89 \mathrm{~b}$ & $88 \mathrm{~b}$ & $74 \mathrm{~b}$ & $72 \mathrm{ab}$ \\
\hline Diurón (2400) & $98 \mathrm{ab}$ & $97 \mathrm{ab}$ & $87 \mathrm{ab}$ & $80 \mathrm{ab}$ \\
\hline Ametrina/2,4-D (1225/650) & $64 \mathrm{c}$ & $60 \mathrm{c}$ & $43 \mathrm{c}$ & $35 \mathrm{c}$ \\
\hline & 81 & 70 & 65 \\
\hline \multicolumn{5}{|l|}{$\begin{array}{l}\text { Tebuthiurón + diurón/ } \\
\text { hexazinona }(1000+\end{array}$} \\
\hline $\begin{array}{l}817,2 / 102,2) \\
\text { Tebuthiurón + diurón }\end{array}$ & $95 \mathrm{ab}$ & $92 \mathrm{~b}$ & $87 \mathrm{ab}$ & $83 \mathrm{ab}$ \\
\hline$(1.000+1.200)$ & $94 \mathrm{~b}$ & $94 \mathrm{ab}$ & $86 \mathrm{ab}$ & $84 a b$ \\
\hline \multicolumn{5}{|l|}{ Tebuthiurón + ametrina/ } \\
\hline 2,4-D (1000 & $86 \mathrm{~b}$ & $82 \mathrm{~b}$ & $70 \mathrm{~b}$ & $64 \mathrm{bc}$ \\
\hline Testigo sin aplicar & $0 \mathrm{~d}$ & $0 \mathrm{~d}$ & $0 \mathrm{~d}$ & $0 \mathrm{~d}$ \\
\hline
\end{tabular}

* Tukey 0,05 .

estadísticas entre tratamientos. El efecto de los herbicidas en la emergencia de plántulas del zacate peludo se observó a partir de los 30 DDA, ya que mientras en el testigo sin aplicar se tenía un promedio de cinco plantas por charola, en el resto de los tratamientos había entre 0,25 y 1,5 plantas por charola, lo que representó una reducción de entre el 70 y $95 \%$ de plántulas emergidas. A los 90 DDA, en el testigo sin aplicar se tenían 6,25 plantas por charola, mientras que en el resto de los

Cuadro 5. Efecto de los tratamientos en el control del complejo de malezas de hoja ancha (\%) a los 15, 30, 60 y 90 DDA. Gabino Barreda, municipio Cosamaloapan, Veracruz, México. 2000.

\begin{tabular}{|c|c|c|c|c|}
\hline Tratamientos (dosis/ha) & 15 DDA & 30 DDA & 60 DDA & 90 DDA \\
\hline Tebuthiurón (1.500) & $100 a^{*}$ & $100 \mathrm{a}$ & $99 \mathrm{a}$ & $98 \mathrm{a}$ \\
\hline \multicolumn{5}{|l|}{ Diurón/hexazinona } \\
\hline$(1.634,4 / 204,3)$ & $100 \mathrm{a}$ & $100 \mathrm{a}$ & $97 \mathrm{ab}$ & $95 \mathrm{ab}$ \\
\hline Diurón (2400) & $99 \mathrm{~b}$ & $98 \mathrm{a}$ & $95 \mathrm{ab}$ & $88 \mathrm{bc}$ \\
\hline Ametrina/2,4-D (1.225/650 & 0) $100 \mathrm{a}$ & 99 a & $88 \mathrm{~b}$ & $83 \mathrm{c}$ \\
\hline \multicolumn{5}{|l|}{$\begin{array}{l}\text { Ametrina/2,4-D + diurón/ } \\
\text { hexazinona }(735 / 390+\end{array}$} \\
\hline \multicolumn{5}{|l|}{ Tebuthiurón + diurón/ } \\
\hline $817,2 / 102,2)$ & $100 \mathrm{a}$ & 99 a & $95 \mathrm{ab}$ & $93 \mathrm{abc}$ \\
\hline $\begin{array}{l}\text { Tebuthiurón + diurón } \\
(1.000+1200)\end{array}$ & $100 \mathrm{a}$ & 99 a & $94 \mathrm{ab}$ & $95 \mathrm{ab}$ \\
\hline \multicolumn{5}{|l|}{ Tebuthiurón + ametrina/ } \\
\hline $2,4-\mathrm{D}(1000$ & $100 \mathrm{a}$ & 99 a & $96 \mathrm{ab}$ & $94 \mathrm{abc}$ \\
\hline Testigo sin aplicar & $0 \mathrm{c}$ & $0 \mathrm{~b}$ & $0 \mathrm{c}$ & $0 \mathrm{~d}$ \\
\hline
\end{tabular}

* Tukey 0,05 
tratamientos se tenían entre 0,25 y 2,25, lo que indicó una reducción en la emergencia de plántulas de entre 64 y $96 \%$. A los 120 DDA, el número de plantas de zacate peludo por charola tuvo un incremento en todos los tratamientos, incluyendo al testigo sin aplicar. Esto pudo deberse a la pérdida de residualidad de los tratamientos herbicidas, o a la producción de semillas en las plantas de zacate peludo, que quedaron en los testigos laterales y que al desprenderse, cayeron sobre el área aplicada y fueron colectadas en el muestreo de esta época. Aun así, en las charolas de los tratamientos herbicidas existió una reducción en el número de plantas de zacate peludo, que varió del 59 al 88\%. Aunque a los 120 DDA no se detectaron diferencias estadísticas entre los tratamientos herbicidas, diurón y ametrina/2,4-D tuvieron el mayor número de plantas de zacate peludo por charola, mientras que en las charolas de diurón/hexazinona, de la mezcla de tebuthiurón + diurón/hexazinona y de tebuthiurón se observó el menor número de ellas. Finalmente, a los 150 DDA, el número de plantas de zacate peludo se incrementó en las charolas del testigo sin aplicar; a su vez, en algunos tratamientos herbicidas se observó un ligero aumento en plantas emergidas, respecto a la evaluación anterior (tratamientos 2, 6 y 8), mientras que en otros hubo una reducción de plantas emergidas (tratamientos 1, 3, 4, 5 y 7). Sin embargo, en todos los tratamientos herbicidas hubo una reducción de entre 79,5 y $91 \%$. de plantas emergidas, con respecto a las del testigo sin aplicar. Al comparar el número de semillas viables de zacate peludo a los 0 y 150 DDA, en todos los tratamientos herbicidas se observó una disminución en el número de semillas viables (se consideraron viables las semillas que germinaron hasta 75 días después de los respectivos muestreos), lo que sugiere que el uso continuo de estos herbicidas puede ser eficiente para lograr reducir el banco de semillas del zacate peludo en los terrenos cañeros. Por otra parte, el no eliminar al zacate peludo aumentó en más del $100 \%$ la cantidad de semillas viables de esta maleza, lo que ocasionará mayor población de esta especie y por ende mayor competencia con el cultivo (Cuadro 6).

Es importante indicar, que si bien, ametrina/2,4-D no tuvo un buen control del zacate peludo que se encontraba emergido al momento de la aplicación, si afectó significativamente la emergencia de nuevas plántulas, por lo que puede ser un buen tratamiento preemergente. Sin embargo, si el zacate ya está emergido, las mejores opciones son las mezclas de tebuthiurón + diurón/hexazinona a $1.000+816 / 102,2 \mathrm{~g} /$ ha y tebuthiurón + diurón a $1.000+1.200 \mathrm{~g} / \mathrm{ha}$.

No se observó toxicidad en las plantas de caña de azúcar aplicadas con tebuthiurón, diurón o la mezcla de ambos herbicidas. En el resto de los tratamientos, hubo una ligera clorosis y necrosis, que afectó entre el 0,5 y
Cuadro 6. Plantas de zacate peludo emergidas de muestras de suelo tomadas a los 0, 30, 90, 120 y 150 DDA. Gabino Barreda, municipio Cosamaloapan, Veracruz. 2000.

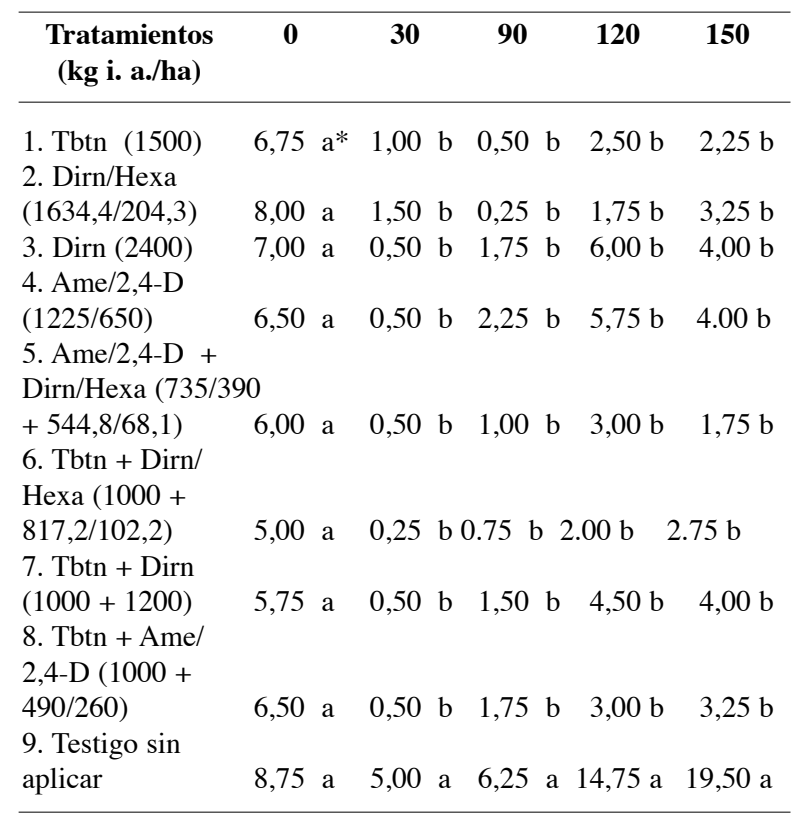

Tbtn $=$ Tebuthiurón, Dirn $=$ Diurón, Hexa $=$ Hexazinona, Ame $=$ Ametrina

* Tukey 0,05 .

el $1 \%$ del área foliar, por lo cual todos los herbicidas evaluados se consideraron altamente selectivos a la caña de azúcar.

\section{CONCLUSIONES}

Tebuthiurón a $1.500 \mathrm{~g} / \mathrm{ha}$ es un tratamiento muy eficiente para el control preemergente del zacate peludo.

Las mezclas postemergentes de tebuthiurón + diurón a $1.000+1.200 \mathrm{~g} / \mathrm{ha}$ y tebuthiurón + diurón/hexazinona a $1.000+817,2 / 102,2 \mathrm{~g} /$ ha proporcionan un control aceptable de esta especie.

Ametrina/2,4-D, solo y en mezcla con tebuthiurón y con diurón/hexazinona no es eficiente para controlar el zacate peludo en postemergencia.

El complejo de malezas de hoja ancha es controlado eficientemente por la mayoría de los tratamientos herbicidas evaluados.

El tebuthiurón, sólo y en mezcla con otros herbicidas residuales puede ser utilizado para reducir la cantidad de semillas viables del zacate peludo en los terrenos cañeros. 


\section{LITERATURA CITADA}

ALTAMIRANO, L.; HERNÁNDEZ, J.; LÓPEZ, A. 2000. Evaluación de la toxicidad del herbicida Sinerge 500 CE en cuatro variedades de caña de azúcar (Saccharum officinarum L.). Tesis Ingeniero Agrónomo. Xalapa, Ver., México. Universidad Veracruzana. Facultad de Ciencias Agrícolas. 78 p.

CASTILLO, A.; MARTÍNEZ, E.; MORGADO, J. 1991. Evaluación de terbutilazina + glifosato 525 en el control de caminadora Rottboellia exaltata en el cultivo de plátano. Papaloapan, Oax. In: ASOMECIMA (ed.). Memoria del XII Congreso Nacional de la Ciencia de la Maleza. Acapulco, Gro., México. 89 p.

ESQUEDA, V. A. 1997. Evaluación de dos formulaciones de tebuthiurón en el control de malezas en el cultivo de caña de azúcar. In: I. Aguilar (ed.). Memorias del XVIII Congreso Nacional de la Ciencia de la Maleza. Cuernavaca, Mor., México. p. 44.

ESQUEDA, V. A. 1999. Control de malezas en caña de azúcar con clomazone y ametrina. Agronomía Mesoamericana 10:23-30.

ESQUEDA, V. A. 2000a. Evaluación de tebuthiurón en mezcla con herbicidas postemergentes en el control de maleza en caña de azúcar (Saccharum officinarum L.). In: F. Guevara (ed.). Memoria del XXI Congreso Nacional de la Ciencia de la Maleza. Morelia, Mich., México. p. 68-73.

ESQUEDA, V. A. 2000b. Las malezas del cultivo del arroz (Oryza sativa L.) en México. Revista Mexicana de la Ciencia de la Maleza. Núm. Especial. p. 63-81.

GÓMEZ, M. A. 1985. Rottboellia exaltata L. f. Gramineae. Una nueva maleza en el cultivo de la caña de azúcar en la región del Papaloapan. México. Instituto para el Mejoramiento de la Producción de Azúcar. Centro Nacional de Investigaciones Azucareras. 11 p.

GOMEZ, K. A.; GOMEZ, A. A. 1984. Statistical procedures for agricultural research. $2^{\text {nd }}$. ed. New York, J. Wiley \& Sons. 680 p.
LENCSE, R.; GRIFFIN, J. 1991. Itchgrass (Rottboellia cochinchinensis) interference in sugarcane (Saccharum sp.). Weed Technol. 5:396-399.

LEÓN, R.; AGÜERO, R. 2001. Efecto de la profundidad del suelo en Rottboellia cochinchinensis (Lour.) Clayton en caña de azúcar (Saccharum officinarum L.). Agron. Mesoamericana 12:65-69.

MEDINA, J. L.; DOMÍNGUEZ, J. A. 2001. Rottboellia cochinchinensis en México: una maleza fuera de la ley. Revista Mexicana de la Ciencia de la Maleza 1:15-18.

MILLHOLLON, R.; BURNER, D. 1993. Itchgrass (Rottboellia cochinchinensis) biotypes in world populations. Weed Sci. 41:379-387.

MORALES, M. D. 1987. Manual de malezas. Instituto para el mejoramiento de la producción de azúcar. Córdoba, Ver., México. Centro Nacional de Investigaciones Azucareras. $51 \mathrm{p}$.

ROJAS, E.; MERAYO, A.; CALVO, G. 1994. La profundidad y duración en el suelo de la semilla de caminadora [Rottboellia cochinchinensis (Lour.) W. D. Clayton] y su efecto sobre la viabilidad y persistencia en el trópico seco. Manejo Integrado de Plagas 32:25-29.

SÁNCHEZ, N; ZÁRATE, R. 2000. Efectividad biológica del herbicida Sencor Duo (metribuzín más 2,4-D) en postemergencia a la maleza en caña de azúcar. In: F. Guevara (ed.). Memoria del XXI Congreso Nacional de la Ciencia de la Maleza. Morelia, Mich., México. p. 178180.

TUCUCH, F. M. 1991. Control químico preemergente de zacate peludo Rottboellia cochinchinensis. In: ASOMECIMA (ed.). Memoria del XII Congreso nacional de la ciencia de la maleza. Acapulco, Gro. México. 83 p.

VILLASEÑOR, J. L.; ESPINOSA, F. J. 1998. Catálogo de Malezas de México. Universidad Nacional Autónoma de México. Consejo Nacional Consultivo Fitosanitario. Fondo de Cultura Económica. México. 449 p. 